\title{
Ion-exchange fibers and drugs: an equilibrium study
}

\author{
Tarja Jaskari $^{\mathrm{a}, \mathrm{b}, *}$, Marja Vuorio $^{\mathrm{c}}$, Kyösti Kontturi ${ }^{\mathrm{c}}$, José A. Manzanares ${ }^{\mathrm{d}}$, \\ Jouni Hirvonen ${ }^{\mathrm{a}}$ \\ ${ }^{\mathrm{a}}$ Department of Pharmacy, Pharmaceutical Technology Division, University of Helsinki, P.O. Box 56, University of Helsinki, \\ FIN-00014 Helsinki, Finland \\ ${ }^{\mathrm{b}}$ Department of Pharmaceutics, University of Kuopio, P.O. Box 1627, FIN-70211 Kuopio, Finland \\ ${ }^{\mathrm{c}}$ Laboratory of Physical Chemistry and Electrochemistry, Helsinki University of Technology, P.O. Box 6100, FIN-02015 TKK, Helsinki, \\ Finland \\ ${ }^{\mathrm{d}}$ Department of Thermodynamics, University of Valencia, E-46100 Burjasot, Spain
}

Received 31 August 2000; accepted 10 November 2000

\begin{abstract}
The purpose of this study was to investigate the mechanisms of drug binding into and drug release from cation-exchange fibers in vitro under equilibrium conditions. Ion-exchange groups of the fibers were weakly drug binding carboxylic acid groups $(-\mathrm{COOH})$, strongly drug binding sulphonic acid groups $\left(-\mathrm{SO}_{3} \mathrm{H}\right)$, or combinations thereof. Parameters determining the drug absorption and drug release properties of the fibers were: (i) the lipophilicity of the drug (tacrine and propranolol are lipophilic compounds, nadolol is a relatively hydrophilic molecule), (ii) the ion-exchange capacity of the fibers, which was increased by activating the cation-exchange groups with $\mathrm{NaOH}$, (iii) the ionic strength of the extracting salt $(\mathrm{NaCl})$, which was studied in a range of $1.5 \mathrm{mM}$ to $1.5 \mathrm{M}$, and finally (iv) the effect of divalent calcium ions $\left(\mathrm{CaCl}_{2}\right)$ on the release of the model drugs, which was tested and compared to monovalent sodium ions $(\mathrm{NaCl})$, and combinations thereof. It was found that the lipophilic drugs, tacrine and propranolol, were retained in the fibers more strongly and for longer than the more hydrophilic nadolol. The more hydrophilic nadolol was released to a greater extent from the fibers containing strong ion-exchange groups $\left(-\mathrm{SO}_{3} \mathrm{H}\right)$, whereas the lipophilic drugs were attached more strongly to strong ion-exchange groups and released more easily from the weak $(-\mathrm{COOH})$ ion-exchange groups. The salt concentration and the choice of the salt also had an effect: at lower $\mathrm{NaCl}$ concentrations more drug was released as a result of the influence of both electrostatic and volume effects (equimolar drug:salt ratio). Incorporation of $\mathrm{CaCl}_{2}$ in the bathing solution increased drug release considerably as compared to $\mathrm{NaCl}$ alone. The equilibrium distribution of the drug species between the fiber and external solution phases was also simulated and it was found that the theoretical modelling proposed describes adequately the basic trends of the behavior of these systems. (c) 2001 Elsevier Science B.V. All rights reserved.
\end{abstract}

Keywords: Ion-exchange fiber; Drug release; Ionic strength; Theoretical modelling

*Corresponding author. Present address: Department of Pharmacy, Pharmaceutical Technology Division, University of Helsinki, P.O. Box 56, University of Helsinki, FIN-00014 Helsinki, Finland. Tel.: + 358-9-1915-9141; fax: +358-9-1915-9144.

E-mail address: tarja.jaskari@helsinki.fi (T. Jaskari).

\section{Introduction}

The achievement of controlled drug delivery and stability of drugs during storage is also a problem for transdermal drug delivery applications $[1,2]$. Adams 
and Holmes synthesized the first ion-exchange resins in 1935 [3]. From 1950 to the present the complexation of drugs with ion-exchange resins has been studied extensively [4-8]. Many drugs are in charged form at the physiological $\mathrm{pH}$ 7.4. The advantage of ion-exchange materials for controlled transdermal drug delivery is their ability to bind and exchange charged drug molecules. A major interest in the use of ion-exchange fibers in formulation development is to provide a controlled release profile and predetermined drug absorption from the transdermal drug delivery system. Recently, our group studied the suitability of cation- and anion-exchange fibers for controlled transdermal drug delivery [9]. The results showed that ion-exchange fibers might be capable of controlling the release rate of drugs and (iontophoretic) transdermal drug delivery across the skin.

Ion exchangers consist of a framework, the socalled matrix, carrying a positive or negative electric fixed charge, which is compensated by mobile counterions of opposite sign. A small amount of mobile ions of the same sign (coions) can also be present. Charged groups bound to the matrix are for instance $-\mathrm{SO}_{3}^{-},-\mathrm{COO}^{-},-\mathrm{PO}_{3}^{2-},-\mathrm{AsO}_{3}^{2-}$ in cation exchangers and $-\mathrm{NH}_{3}^{+},-\mathrm{NH}_{2}^{+},-\mathrm{NH}^{+}$and $-\mathrm{S}^{+}$in anion exchangers [10]. The ion exchange is a stoichiometric process in which any counterions that leave the ion-exchanger are replaced by an equivalent amount of other counterions [11]. This is a consequence of the electroneutrality requirement. The ion exchange is essentially a diffusional process, but also is related to chemical reaction kinetics. Usually the ion exchangers are selective: they take up some counterions in preference to others. The rate-determining step in ion exchange is diffusion either within the ion exchanger itself or in the diffusion boundary layer [10].

Biological factors of transdermal drug delivery, which are (in general) less easily controlled, include intra- and intersubject variability, regional blood flow, and skin pH [12]. Better control of biological variability in transdermal drug absorption has been attempted with ion-exchange systems. Selection of external conditions (e.g. ionic strength, $\mathrm{pH}$, and choice of the salt in the release medium), drug properties (charge, lipophilicity and molecular weight), and fiber quality (ion-exchange groups, capacity, grafting), may be utilized to control the release kinetics of a drug from ion-exchange systems [7-9]. In the present study, the effects of these factors on the absorption and release of three model drugs was studied theoretically and experimentally. We analyzed the effect of (i) lipophilicity of the drugs, (ii) cation-exchange fiber activation with $\mathrm{NaOH}$, (iii) ionic strength of the extracting salt at equimolar concentration with the drug, and (iv) divalent calcium ions compared to monovalent sodium ions, and their combination. Determination of the equilibrium distribution of the drug species between the fiber and external solution phases was the major objective of this study.

\section{Materials and methods}

\subsection{Chemicals}

The positively charged ( $\mathrm{pH}$ 7.4) model drugs studied, tacrine $(-\mathrm{HCl})$, propranolol $(-\mathrm{HCl})$ and nadolol were from Sigma (St. Louis, MO, USA). Extracting salts, buffers and other chemicals were of analytical grade and were used without further purification. Deionized (Millipore ${ }^{\mathrm{TM}}$ ) Milli-Q water (resistivity $\geq 18 \mathrm{M} \Omega / \mathrm{cm}$ ) was used to prepare all solutions. The ion-exchange fibers Smopex ${ }^{\circledR}-101[-$ $\mathrm{SO}_{3} \mathrm{H}$ ion-exchange groups, poly(ethylene- $g$ styrenesulphonicacid) fiber], Smopex ${ }^{\circledR}-102$ [-COOH ion-exchange groups, poly(ethylene- $g$-acrylicacid) fiber] and Smopex ${ }^{\circledR}-107$ [1:1 $-\mathrm{COOH}$ and $-\mathrm{SO}_{3} \mathrm{H}$ ion-exchange groups, poly(ethylene- $g$-acrylicacidco-vinylsulphonicacid)fiber] were obtained from SmopTech (Turku, Finland). Maximal ion-exchange capacity of the 101-fiber was $3.2 \mathrm{mmol} / \mathrm{g}$, of the 102 -fiber $8.0 \mathrm{mmol} / \mathrm{g}$, and of the 107-fiber 8.0 $\mathrm{mmol} / \mathrm{g}$.

\subsection{Preparation of the drug containing ion- exchange fiber discs}

Circular discs (diameter $15 \mathrm{~mm}$ ) were cut from the cation-exchange fibers. The weight of the discs was 50-100 mg depending on the fiber: the thickness of the Smopex ${ }^{\circledR}-107$ fiber was $\sim 3 \mathrm{~mm}$, the Smopex ${ }^{\circledR}$ 102 fiber was $\sim 6 \mathrm{~mm}$, and the Smopex ${ }^{\circledR}-101$ fiber was like a cotton cloth. To activate the ion-exchange 
groups, the fiber discs were treated with $0.1 \mathrm{M} \mathrm{NaCl}$ solution or $0.1 \mathrm{M} \mathrm{NaCl} / 0.1 \mathrm{M} \mathrm{NaOH}$ (1:1) solution for $\sim 30 \mathrm{~min}$. Thereafter, the fiber discs were washed with purified water. The discs were immersed in a $1 \%(\mathrm{~m} / \mathrm{V})$ tacrine $(-\mathrm{HCl})$, propranolol $(-\mathrm{HCl})$ or nadolol solution three times consecutively. The first and second times the discs were immersed for $3 \mathrm{~h}$ and the third time overnight $(\sim 12 \mathrm{~h})$. After each immersion, the discs were washed with purified water. The amounts of absorbed drugs in the fiber discs were determined by HPLC from the combined washing solutions.

\subsection{Drug release from the ion-exchange fiber in vitro}

Drug release from the cation-exchange fiber discs was tested in vitro in glass dishes (with bottle top) at a temperature of $25^{\circ} \mathrm{C}$. Drug containing fiber discs were separately placed in $\mathrm{NaCl}$ solutions $(0.0015$, $0.015,0.15$ and $1.5 \mathrm{M})$. The redistribution of the drug between the ion-exchanger phase and the bathing solution depends not only on the external solution concentration but also on the solution volume. A disc placed in a large volume of a concentrated electrolyte solution would exchange practically all the drug with the solution, and no conclusion could be drawn about the tendency of the drug leave the fiber under these conditions. To avoid this problem, different volumes of the release medium were used. The volumes were chosen so that each $\mathrm{NaCl}$ solution contained an equimolar concentration of the salt as the concentration of the drug that was in the fiber. To measure drug release from the fiber, the $\mathrm{NaCl}$ solutions were changed five times during a week (at 1, 2, 3, 4, and 7 days). The results are shown in Figs. 1-4 for equilibration stages 1-5, respectively. Fiber discs were washed with purified water $(10 \mathrm{ml})$ and squeezed, the washing solutions were collected, and the released drug concentrations in these solutions were determined by HPLC.

In addition to drug release tests (three model drugs) in $\mathrm{NaCl}$, tacrine release from the Smopex ${ }^{\circledR}$ $101,-102$ and -107 fibers was tested in the presence of $10 \% / 90 \%, 50 \% / 50 \%$ and $90 \% / 10 \%(\mathrm{~m} / \mathrm{V})$ $\mathrm{CaCl}_{2} / \mathrm{NaCl}$ solutions. The total $\mathrm{NaCl}+\mathrm{CaCl}_{2}$ concentration was $0.015 \mathrm{M}$ in each case. In these experiments, the fibers were activated with $0.1 \mathrm{M}$ $\mathrm{NaCl} / 0.1 \mathrm{M} \mathrm{NaOH}$ solution.

\subsection{Analysis of the drugs}

The drugs were analysed by HPLC (Beckman System Gold, Beckman Instruments, San Ramon, CA, USA). Tacrine, propranolol and nadolol were analyzed with a supelcosil LC-18-DB column (150X $4.6 \mathrm{~mm}, 5 \mu \mathrm{m}$; Supelco, USA). The flow rate was $1.0 \mathrm{ml} / \mathrm{min}$ in each case. Analysis methods were similar to our previous study [9]. For tacrine, the mobile phase included $22 \%$ acetonitrile, $1 \%$ triethylamine, and $77 \%$ deionized water at $\mathrm{pH} 6.5(\lambda=240$ $\mathrm{nm})$. For propranolol analysis the mobile phase was $35 \%$ acetonitrile and $65 \%$ acetate buffer at $\mathrm{pH} 4.0$ $(\lambda=289 \mathrm{~nm})$. Nadolol was analyzed with $25 \%$ acetonitrile, $1 \%$ heptane sulphonic acid, and $74 \%$ acetate buffer at $\mathrm{pH} 4.0(\lambda=223 \mathrm{~nm})$.

\section{Theory}

\subsection{Drug partition equilibrium}

The equilibrium distribution of the drug species between the fiber and external solution phases results from both electrostatic and hydrophobic interactions. The former are measured by the electrical partition coefficient $[10,13]$

$K_{\mathrm{e}, \mathrm{d}}=\exp \left(-z_{\mathrm{d}} F \phi_{\mathrm{D}} / R T\right)$

where $F, R$, and $T$ are the Faraday constant, the gas constant, and the thermodynamic temperature, respectively, and $\phi_{\mathrm{D}} \equiv \bar{\phi}-\phi$ (i.e. the electrical potential in the fiber with respect to the external phase) is the Donnan potential. Overbars denote fiber phase throughout this study. The Donnan potential is determined by the ion-exchange capacity of the fiber and the nature and concentration of the external solution. In the case of cationic drugs (charge number $z_{\mathrm{d}}>0$ ) in cation-exchange membranes, $\phi_{\mathrm{D}}$ is negative and $K_{\mathrm{e}, \mathrm{d}}>1$.

The chemical partition coefficient [14]

$K_{\mathrm{c}, \mathrm{d}}=\exp \left[\left(\mu_{\mathrm{d}}^{0}-\bar{\mu}_{\mathrm{d}}^{0}\right) / R T\right]$

measures the tendency of the drug to get into the 


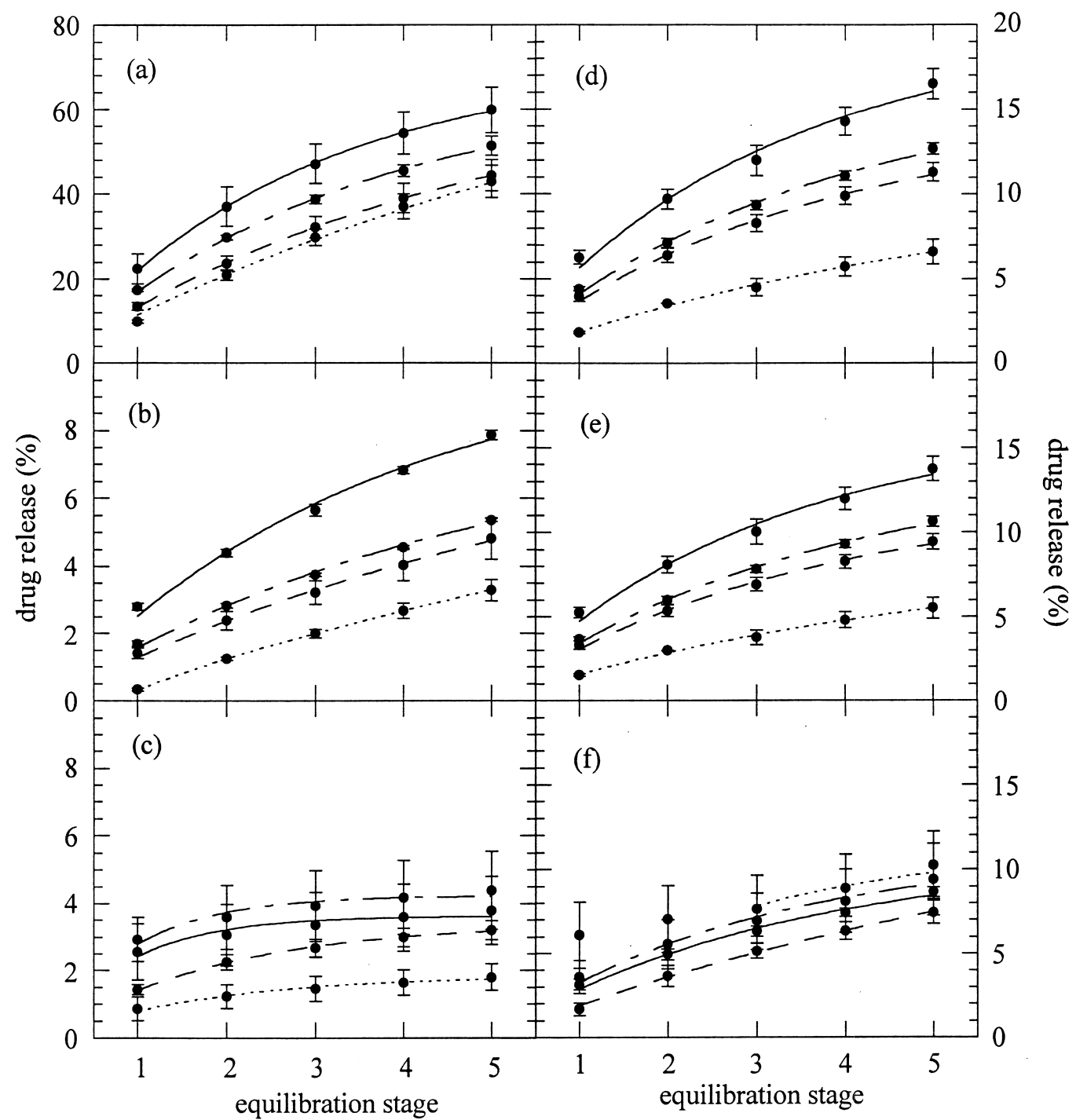

Fig. 1. Effect of electrolyte concentration on the release of nadolol (a,d), propranolol (b,e), and tacrine (c,f) from sulphonic acid $\left(\right.$ Smopex $\left.{ }^{\circledR}-101\right)(\mathrm{a}, \mathrm{b}, \mathrm{c})$ and carboxylic acid $\left(\right.$ Smopex $\left.^{\circledR}-102\right)$ fibers $(\mathrm{d}, \mathrm{e}, \mathrm{f}):(-) 1.5 \mathrm{mM},\left(--{ }_{-}\right) 15 \mathrm{mM},(---) 0.15 \mathrm{M},(-----) 1.5$ M. Smooth fitting curves to the experimental data have been drawn to facilitate the interpretation of the figure.

hydrophobic fiber as a result of its specific interaction with the fiber. In Eq. (2), $\mu_{\mathrm{d}}^{0}$ is the standard chemical potential of the drug species. The more hydrophobic the drug, the larger the decrease in free energy associated with its interaction with the fiber and the larger the value of $K_{\mathrm{c}, \mathrm{d}}(>1)$. In the case of hydrophilic drugs, however, $K_{\mathrm{c}, \mathrm{d}}$ could be $<1$. (The water content of the fiber also affects the value of $K_{\mathrm{c}, \mathrm{d}}$.)
As a result, the molar concentration ratio of the drug is $[13,15]$

$\frac{\bar{c}_{\mathrm{d}}}{c_{\mathrm{d}}}=K_{\mathrm{p}, \mathrm{d}}=K_{\mathrm{e}, \mathrm{d}} K_{\mathrm{c}, \mathrm{d}}$.

This equation states that the electrochemical potential of the drug species takes the same value in the fiber and external solution phases under equilibrium conditions (Donnan equilibrium). Note that the drug 


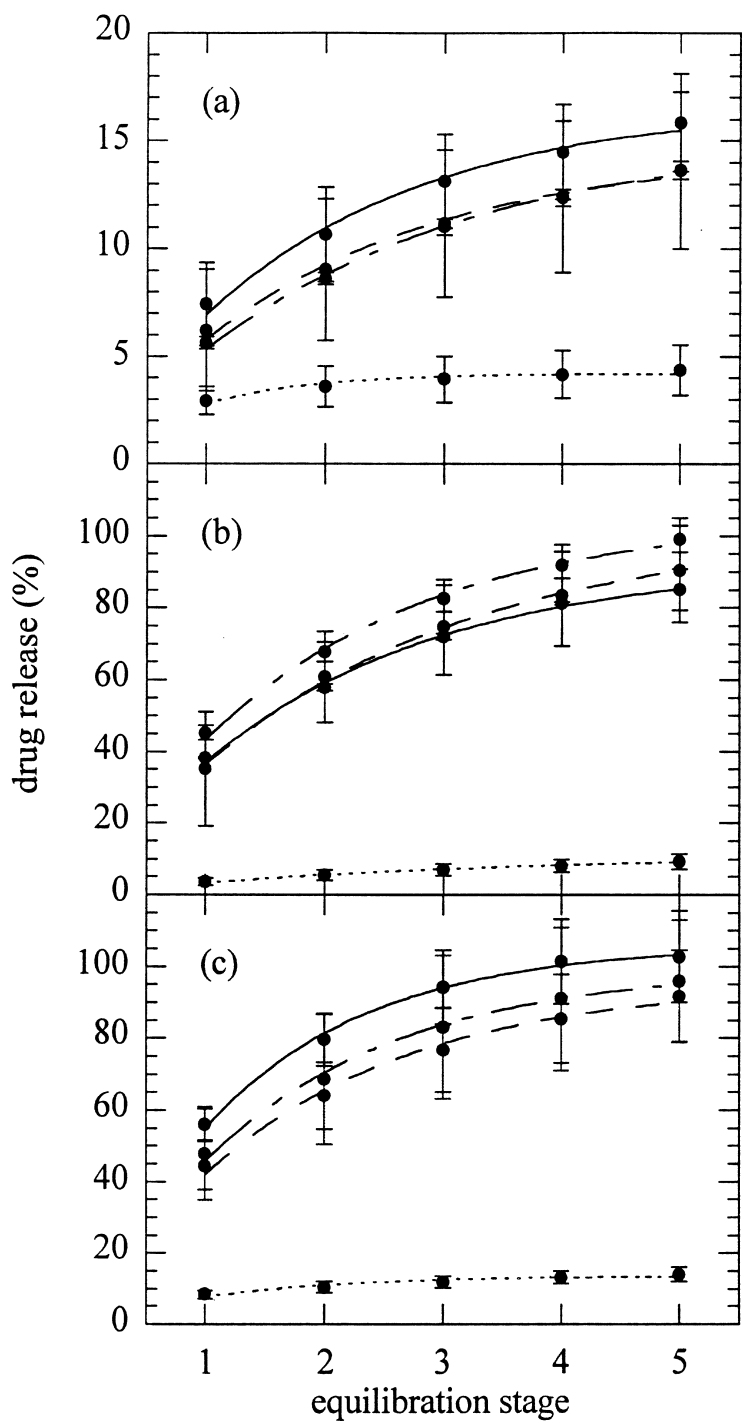

Fig. 2. Effect of calcium ions on the release of tacrine from Smopex ${ }^{\circledR}-101$ (a, 100\% sulphonic acid), -102 (b, 100\% carboxylic acid), and -107 (c, 50\%/50\% sulphonic/carboxylic acid) fibers: (-) 90\%, (- - ) 50\%, (- - ) 10\%, (- - -) 0\% $\mathrm{CaCl}_{2}$. Total concentration of $\mathrm{NaCl}+\mathrm{CaCl}_{2}$ was $15 \mathrm{mM}$. Smooth fitting curves to the experimental data have been drawn to facilitate the interpretation of the figure.

concentration is relatively low in both phases, and therefore no activity coefficients have been included.

\subsection{The effect of ionic strength on drug release}

In the experimental procedure, the fiber discs were

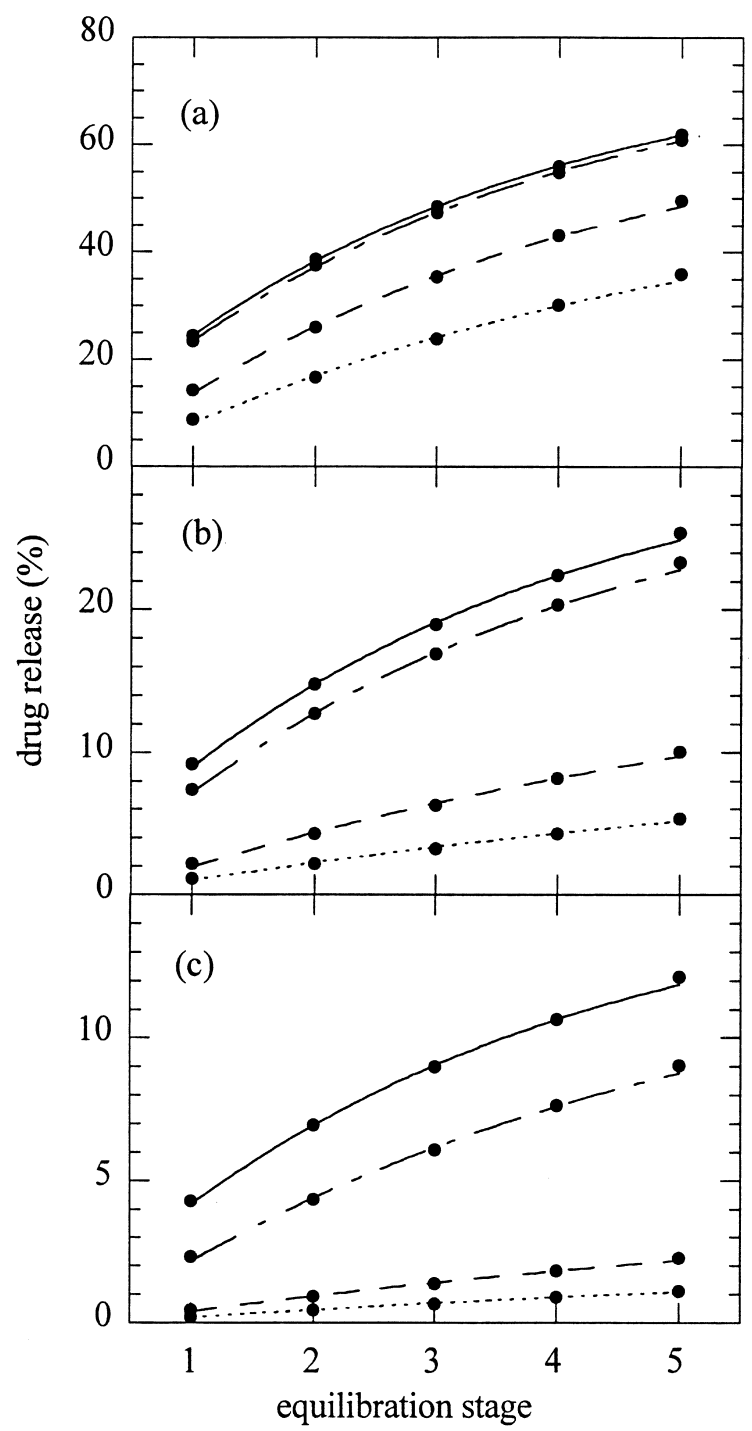

Fig. 3. Effect of electrolyte concentration on the release for different values of drug lipophilicity $K_{\mathrm{c}, \mathrm{d}}=10$ (a), 100 (b), and 500 (c): (—) $1.5 \mathrm{mM},(---) 15 \mathrm{mM},(---) 0.15 \mathrm{M},\left(---_{-}\right)$ $1.5 \mathrm{M}$.

initially immersed several times in a $1 \%(\mathrm{~m} / \mathrm{V})$ drug solution and washed with purified water. The discs prepared in this way contained practically no chloride ions. The amount of drug inside the fiber disc, $n_{\mathrm{d}}^{0}$, was accurately determined from the washing solutions and, therefore, the initial drug concentration inside the fiber is $\bar{c}_{\mathrm{d}}^{0}=n_{\mathrm{d}}^{0} / \bar{V}$, where $\bar{V}$ is the fiber volume. Moreover, the local electroneutrality 


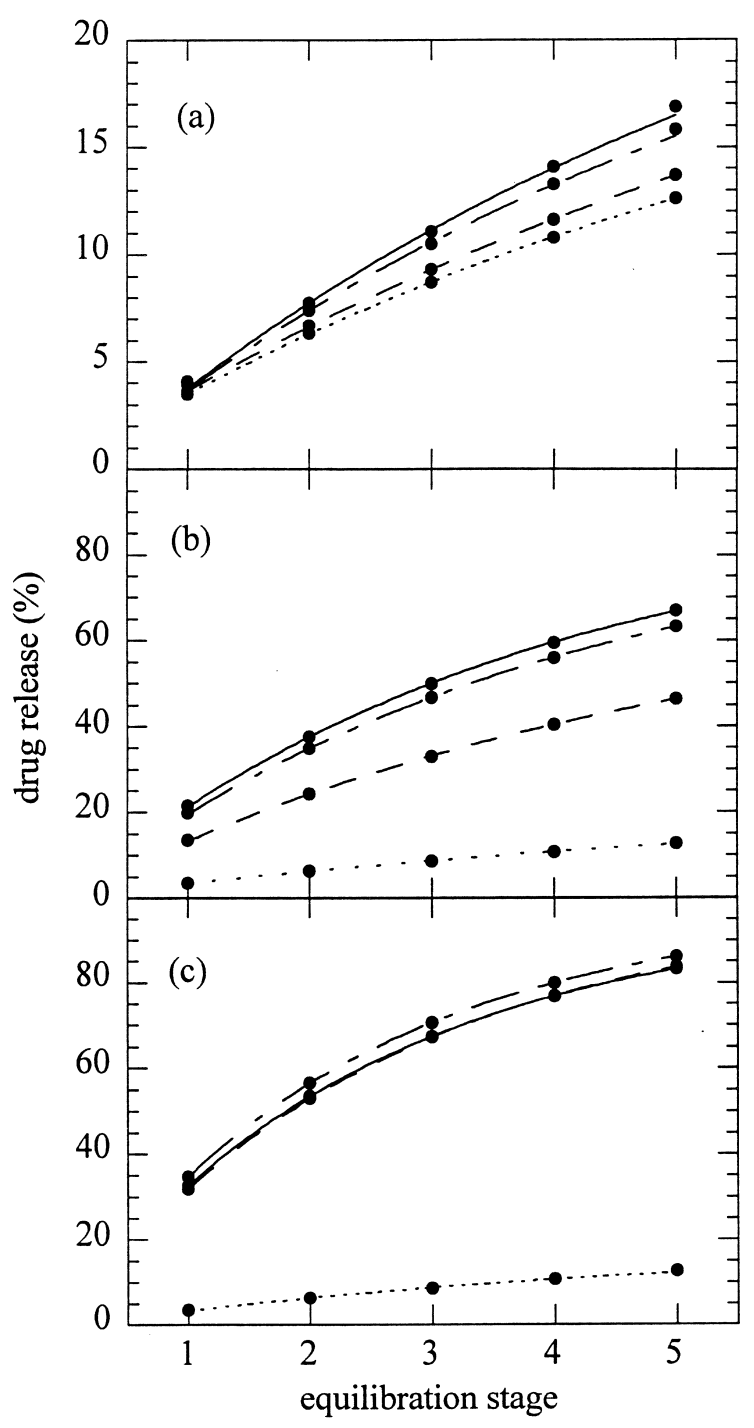

Fig. 4. Effect of calcium ions on the release for $K_{\mathrm{c}, \mathrm{d}}=300$ and different values of the binding constant $K_{\mathrm{b}}=0$ (a), $1 \mathrm{mM}$ (b), and $50 \mathrm{mM}$ (c): (-) 90\%, (- - ) 50\%, (- - ) 10\%, (- - -) $0 \% \mathrm{CaCl}_{2}$. Total concentration of $\mathrm{NaCl}+\mathrm{CaCl}_{2}$ was $15 \mathrm{mM}$.

condition requires that the concentration of ion-exchange groups bound to the fiber discs is $c_{m}=\left|z_{\mathrm{d}}\right|$ $z_{m} \mid \bar{c}_{\mathrm{d}}^{0}$, where $z_{m}$ denotes the charge number of these groups.

Then the discs were introduced into electrolyte solutions. The volume $V$ used of the electrolyte solution was inversely proportional to its concentration, so that the number of moles of external electrolyte cations $n_{+}^{0}=c_{+}^{0} V$

was always the same. The limited amount of electrolyte ions in the partitioning equilibria, causes the drug release to be influenced by both electrostatic and volume effects.

While the partition equilibrium of the drug is highly influenced by the specific (chemical) interactions, the partition equilibrium of the electrolyte ions is mostly dictated by electrostatic interactions and, hence, their partition coefficient is simply given by $[13,15]$

$\frac{\bar{c}_{i}}{c_{i}}=K_{\mathrm{e}, i}=K_{\mathrm{e}}^{-z_{i}}$

where $K_{\mathrm{e}}=\exp \left(F \phi_{\mathrm{D}} / R T\right)$ and $z_{i}$ is the charge number of species $i$. Considering that the drug solution is completely dissociated, the Donnan potential, $\phi_{\mathrm{D}}$, is determined by the local electroneutrality conditions in the fiber and external solutions [10]

$$
\begin{aligned}
& -z_{m} c_{m}=z_{\mathrm{d}} \bar{c}_{\mathrm{d}}+z_{+} \bar{c}_{+}+z_{-} \bar{c}_{-} \\
& \quad=z_{\mathrm{d}} c_{\mathrm{d}} K_{\mathrm{c}, \mathrm{d}} K_{\mathrm{e}}^{-z_{\mathrm{d}}}+z_{+} c_{+} K_{\mathrm{e}}^{-z_{+}}+z_{-} c_{-} K_{\mathrm{e}}^{-z_{-}} \\
& 0=z_{\mathrm{d}} c_{\mathrm{d}}+z_{+} c_{+}+z_{-} c_{-} .
\end{aligned}
$$

In the simple case $z_{\mathrm{d}}=z_{+}=1, z_{-}=z_{m}=-1$ (i.e. 1:-1 electrolyte solution, univalent cationic drug and cation-exchange fiber), Eqs. (6) and (7) lead to

$$
\begin{aligned}
& \left(c_{\mathrm{d}} K_{\mathrm{c}, \mathrm{d}}+c_{+}\right) K_{\mathrm{e}}^{-1}-\left(c_{\mathrm{d}}+c_{+}\right) K_{\mathrm{e}}-c_{m}=0 \\
& K_{\mathrm{e}}=\frac{-c_{m}+\left[c_{m}^{2}+4\left(c_{+}+c_{\mathrm{d}}\right)\left(c_{+}+c_{\mathrm{d}} K_{\mathrm{c}, \mathrm{d}}\right)\right]^{1 / 2}}{2\left(c_{+}+c_{\mathrm{d}}\right)} .
\end{aligned}
$$

It is important to observe that concentrations $c_{\mathrm{d}}$ and $c_{+}$depend on $K_{\mathrm{e}}$, because the limited amount of both the drug and external electrolyte ions requires that the conditions

$n_{\mathrm{d}}^{0}=c_{\mathrm{d}} V+\bar{c}_{\mathrm{d}} \bar{V}$

$n_{+}^{0}=c_{+} V+\bar{c}_{+} \bar{V}$

must be satisfied. Note also from Eq. (9) that $\phi_{\mathrm{D}}=$ $(R T / F) \ln K_{\mathrm{e}}$ may take positive values (i.e. $K_{\mathrm{e}}>1$ ) when $K_{\mathrm{c}, \mathrm{d}} \gg 1$. In other words, the fiber might 
behave as an anion-exchange fiber due to strong chemical interactions between the drug and the fiber, and in this case the effect of increasing electrolyte concentration would be to reduce the drug release.

After equilibration, the discs were squeezed, thus reducing their volume in $\Delta V$. This process eliminates a neutral solution composed by a large fraction of the coions and the same amount of counterions. If we denote by $\Delta n_{i}$ the number of moles of species $i$ that are squeezed out of the fiber, the concentration of species $i$ in the fiber $\left(\bar{c}_{i}^{\prime}\right)$ and in the solution $\left(c_{i}^{\prime}\right)$ after squeezing are, respectively,

$\bar{c}_{i}^{\prime}=\frac{\bar{c}_{i} V-\Delta n_{i}}{V-\Delta V}$

$c_{i}^{\prime}=\frac{c_{i} V+\Delta n_{i}}{V+\Delta V}$.

Assuming that Eqs. (3) and (5) are still valid after squeezing, although with a different value of $K_{\mathrm{e}}$, Eq. (6) can be used to determine the values of $\Delta n_{i}$.

The whole procedure was repeated five times (after 1, 2, 3, 4, and 7 days), and the situations achieved are denoted as equilibrium stages 1-5, respectively. It is admitted that the equilibrium distribution has been reached after 1 day and, therefore, that the equilibrium Eqs. (11a) and (11b) can be used to simulate the concentrations that would be experimentally measured at those times.

\subsection{Bathing solutions containing calcium ions}

Calcium ions are known to adsorb strongly to carboxylic groups, but not to sulphonic groups $[15,16]$. The behavior of fibers with these two types of ion-exchange groups is, therefore, expected to be different when calcium ions are present in the external electrolyte solution, as was confirmed by the experiments.

The adsorption of a $\mathrm{Ca}^{2+}$ ion to a $\mathrm{COO}^{-}$group generates a group $\mathrm{COO} \cdots \mathrm{Ca}^{+}$, which acts as an anion-exchange group. When this adsorption is strong enough, the fiber behaves as an anion-exchange fiber. If $x_{-}$and $x_{+}$denote the molar fractions of carboxylic groups in the negatively $\left(\mathrm{COO}^{-}\right)$and positively $\left(\mathrm{COO} \cdots \mathrm{Ca}^{+}\right)$charged forms, respectively, and $K_{\mathrm{b}}=x_{+} /\left(\bar{c}_{\mathrm{Ca}^{2+}} x_{-}\right)$stands for the binding constant of $\mathrm{Ca}^{2+}$ ions to $\mathrm{COO}^{-}$groups, the net charge associated to the membrane is [17]

$z_{m} c_{m}=\left(x_{+}-x_{-}\right) c_{\mathrm{T}}=\frac{K_{\mathrm{b}} \bar{c}_{\mathrm{Ca}^{2+}}-1}{K_{\mathrm{b}} \bar{c}_{\mathrm{Ca}^{2+}}+1} c_{\mathrm{T}}$

where $c_{\mathrm{T}}$ is the total concentration of fixed groups in the fiber. Note that $z_{m} c_{m}=-c_{\mathrm{T}}$ when $K_{\mathrm{b}} \bar{c}_{\mathrm{Ca}^{2+}} \ll 1$ (weak binding), and $z_{m} c_{m}=c_{\mathrm{T}}$ when $K_{\mathrm{b}} \bar{c}_{\mathrm{Ca}^{2+}} \gg 1$ (strong binding).

When using mixed $\mathrm{NaCl} / \mathrm{CaCl}_{2}$ solutions, the Donnan potential $\phi_{\mathrm{D}}=(R T / F) \ln K_{\mathrm{e}}$ is given by the local electroneutrality conditions in the fiber and external solutions

$$
\begin{aligned}
& \left(c_{\mathrm{d}} K_{\mathrm{c}, \mathrm{d}}+c_{\mathrm{H}+}+c_{\mathrm{Na}+}\right) K_{\mathrm{e}}^{-1}+2 c_{\mathrm{Ca}^{2+}} K_{\mathrm{e}}^{-2} \\
& \quad-c_{\mathrm{Cl}^{-}} K_{\mathrm{e}}-z_{m} c_{m}=0 \\
& c_{\mathrm{d}}+c_{\mathrm{H}^{+}}+c_{\mathrm{Na}^{+}}+2 c_{\mathrm{Ca}^{2+}}-c_{\mathrm{Cl}-}=0 .
\end{aligned}
$$

The simulation of the drug release experiments is then carried out as described in the previous section, including also the equation $n_{\mathrm{Ca}^{2+}}^{0}=c_{\mathrm{Ca}^{2+}} V+\bar{c}_{\mathrm{Ca}^{2+}} \bar{V}$, which takes into account the finite amount of calcium ions.

\section{Results and discussion}

\subsection{The drug binding capacity of ion-exchange fibers}

The drug binding experiments can be considered as partition equilibrium experiments, where the volume of the external solution is large enough so as to consider the external solution concentration constant. This solution contains the drug in salt form at a concentration $c_{\mathrm{d}}$. The equilibrium concentration of the drug in the fiber $\bar{c}_{\mathrm{d}}$ is obtained from Eqs. (3) and (9), with $c_{+}=0$, as [13]

$\bar{c}_{\mathrm{d}}=\frac{c_{m}}{2}+\left[\left(\frac{c_{m}}{2}\right)^{2}+K_{\mathrm{c}, \mathrm{d}} c_{\mathrm{d}}^{2}\right]^{1 / 2}$.

Table 1 shows the drug content of ion-exchange fibers containing sulphonic groups (Smopex ${ }^{\circledR}-101$ ), carboxylic groups (Smopex ${ }^{\circledR}-102$ and $-102 *$ ) and their combination (Smopex $\left.{ }^{\circledR}-107\right)$. The drugs have been presented in order of increasing lipophilicity as determined from their octanol/water partition coeffi- 
Table 1

Drug content (in $\mathrm{mmol} / \mathrm{g}$ ) in cation-exchange fibers Smopex ${ }^{\circledR}$ 101 (100\% sulphonic acid), -102 (100\% carboxylic acid) and -107 (50\%/50\% sulphonic/carboxylic acid) after $24 \mathrm{~h}$ of equilibration ${ }^{\mathrm{a}}$

\begin{tabular}{|c|c|c|c|c|}
\hline \multirow[t]{2}{*}{ Drug } & \multicolumn{4}{|l|}{ Fiber } \\
\hline & -101 & -102 & $-102 *$ & -107 \\
\hline Nadolol & 1.88 & 2.28 & 4.54 & 0.98 \\
\hline Propranolol & 3.09 & 2.94 & 8.33 & 2.69 \\
\hline Tacrine & 4.43 & 1.43 & 8.30 & 0.98 \\
\hline
\end{tabular}

${ }^{\mathrm{a}}$ The fibers were activated with $0.1 \mathrm{M} \mathrm{NaCl}$ solution or, in the case denoted as $102^{*}$, with $0.1 \mathrm{M} \mathrm{NaCl} / 0.1 \mathrm{M} \mathrm{NaOH}$ solution.

cient [9] (i.e. tacrine and propranolol are more lipophilic and nadolol more hydrophilic). From this table, particularly from the columns corresponding to Smopex ${ }^{\circledR}-101$ and $-102 *$, it can be observed that the drug content increases with increasing lipophilicity (i.e. increasing $K_{\mathrm{c}, \mathrm{d}}$ ) as predicted by Eq. (15). The differences shown in Table 1 in the drug content values for a given drug in different fibers is not really in contradiction with this statement, but it rather is a clear manifestation of the fact that the distribution equilibrium of the drug is affected by drug-fiber interactions, which are specific to the ion-exchange group and the nature of the fiber. The strength of these interactions also affects the value of the chemical partition coefficient, $K_{\mathrm{c}, \mathrm{d}}$.

The drug content $\bar{c}_{\mathrm{d}}$ is always larger than $c_{m}$, and the difference $\bar{c}_{\mathrm{d}}-c_{m}$ is equal to the concentration of coions (i.e. anions) inside the fiber. The actual value of $c_{m}$ in the fiber used in these experiments is not known, but it is expected to be a fraction of the maximum ion-exchange capacity of the fibers, which are $3.2 \mathrm{mmol} / \mathrm{g}$ (Smopex ${ }^{\circledR}-101$ ) and $8.0 \mathrm{mmol} / \mathrm{g}$ (Smopex ${ }^{\circledR}-102$ and 107). Since the values in Table 1 are only slightly larger than the maximum ion-exchange capacity of the fibers, the amount of coions seems to be relatively small.

When the fiber Smopex ${ }^{\circledR}-102$ with weak carboxylic groups was activated with a $\mathrm{NaCl} / \mathrm{NaOH}$ solution (Smopex $\left.{ }^{\circledR}-102 *\right)$, the fixed charge concentration $c_{m}$ reached a value approximately equal to the maximum ion-exchange capacity of this fiber. The increase in drug binding in this case (with respect to Smopex ${ }^{\circledR}-102$ with $\mathrm{NaCl}$ activation) is, therefore, attributed to the increase in $c_{m}$, although the reason why the increase is more pronounced in the case of lipophilic drugs remains unclear.

\subsection{Drug release from the ion-exchange fibers in vitro}

Fig. 1 shows the experimental results on the effect of electrolyte $(\mathrm{NaCl})$ concentration on drug release from fibers Smopex ${ }^{\circledR}-101$ (sulphonic acid) and -102 (carboxylic acid). The first noticeable difference between the release of the drugs from these two fibers is that the drug release values are very different (note the different scales of the ordinate axes). Although the lipophilicity scale tacrine $\approx$ propranolol $>$ nadolol is respected in both cases, in the sense that larger lipophilicity implies lower drug release, the release of nadolol from Smopex ${ }^{\circledR}-101$ is over ten times larger than that of tacrine from this same fiber (compare Fig. 1a,c), while it is only about twice as large in the case of Smopex ${ }^{\circledR}-102$ (compare Fig. 1d,f). This indicates that the specific interaction of the lipophilic drugs propranolol and tacrine with the sulphonic acid fiber is stronger than with the carboxylic acid fiber, while the opposite is true for the interaction of nadolol with these fibers. This conclusion is in full agreement with the first two columns in Table 1. Other researchers have found the same effect, e.g., Sprockel and Price observed that drug is released more quickly from a carboxylic acid resin than from a sulphonic acid resin [18].

Effect of fiber activation with $\mathrm{NaOH}$ upon drug release was also studied. Activation of fibers with $\mathrm{NaOH}$ increased drug binding to ion-exchange groups (Table 1), but the fraction of the drug content released in every case was about the same (data not shown). The amount of tacrine released from the Smopex ${ }^{\circledR}-102$ fiber in the $0.015 \mathrm{M} \mathrm{NaCl}$ solution in 1 week was $(9.40 \pm 2.1) \%$ when the tacrine content in the fiber was $1.43 \mathrm{mmol} / \mathrm{g}$, and $(8.71 \pm 0.85) \%$ with the initial tacrine content of $8.30 \mathrm{mmol} / \mathrm{g}$. Correspondingly, with propranolol the release was $(10.6 \pm 0.3) \%$ at $2.94 \mathrm{mmol} / \mathrm{g}$ drug content and $(12.8 \pm 0.3) \%$ when the drug content was $8.33 \mathrm{mmol} /$ g. Thus, although the drug content in the fibers was increased leading to the greater amount of drug released, the fraction of the drug released remained about constant. Conaghey et al. have observed the same influence with ion-exchange resins, when a 
constant fraction of drug content was released from the resins, although the drug content in the resins was increased [8].

In the cases of nadolol and propranolol, Fig. 1 clearly shows that the drug release increases with decreasing electrolyte concentration. This trend is not as evident in the case of tacrine due to the stronger specific interactions, which lead to very low drug release values. These results can be easily understood from the consideration of Eq. (4), i.e. from the change of the solution volume with electrolyte concentration. The volume $V$ used in the release experiments with $1.5 \mathrm{mM} \mathrm{NaCl}$ was 1000 times larger than in the experiments with $1.5 \mathrm{M}$ $\mathrm{NaCl}$, and this difference in volume has certainly an influence on the drug release, as described by Eqs. (10a) and (10b). The change in electrolyte concentration has two competing effects on the drug release. On one hand, increasing electrolyte concentration decreases the Donnan potential and, hence, the electrostatic affinity between the drug and the fiber, thus tending to increase drug release. On the other hand, a large electrolyte concentration implies a small volume of the external solution and then a very small amount of released drug is needed to reach the equilibrium drug concentration in the external solution. Fig. 1 shows that this latter volume effect is dominant.

The opposite situation (i.e. drug release increasing with increasing electrolyte concentration) would be observed if the same external solution volume were used for the different electrolyte concentrations. This has indeed been observed by other authors with similar ion-exchange systems $[7,19]$.

Since the specific interaction between tacrine and the fibers is so strong that the tacrine release is very small at all electrolyte concentrations (Fig. 1), the release of tacrine was also studied with mixed solutions containing $\mathrm{NaCl}$ and $\mathrm{CaCl}_{2}$ at fixed total concentration. Fig. 2 shows the experimental results regarding the effect of calcium ions on drug release. It was found that even $10 \%$ of $\mathrm{CaCl}_{2}$ had a pronounced effect on the drug release. The release was virtually complete from Smopex ${ }^{\circledR}-107$, which has the lower ion-exchange capacity (according to Table 1 ), while it increased dramatically in the case of fiber Smopex ${ }^{\circledR}-102$, and to a lesser extent in the case of Smopex ${ }^{\circledR}-101$. This effect is due to the binding of calcium ions into the carboxylic groups, which is known to be very strong at the $\mathrm{pH}$ of these experiments [16].

Figs. 3 and 4 show simulations of experiments, which have been carried out assuming a fiber volume $\bar{V}=1 \mathrm{ml}$, squeezed by $\Delta V=0.5 \mathrm{ml}$, and loaded with an initial amount of drug $n_{\mathrm{d}}^{0}=100 \mu \mathrm{mol}$. Similarly to the experimental procedure, only five values have been simulated, corresponding to the equilibrium distributions reached at times $1,2,3,4$, and 7 days (equilibration stages $1-5$, respectively). Continuous lines have been drawn on those values to facilitate the figure interpretation. The effects of the drug lipophilicity and the external electrolyte concentration are considered in Fig. 3. The different scales used in Fig. $3 \mathrm{a}-\mathrm{c}$ clearly show that the increase of $K_{\mathrm{c}, \mathrm{d}}$ leads to a decrease of the drug release, as could be expected. In each of these figures, the increase of the external electrolyte concentration leads to a decrease of the drug release, in agreement with the experimental observations. This effect is more significant (in relative terms) in the case of drugs with moderate to large lipophilicity (i.e. Fig. 3b,c) since in this case the Donnan potential is smaller and the volume effects mentioned above become dominant.

Fig. 4 illustrates the effects of the nature of the external electrolyte solution (ternary solution $\mathrm{NaCl} /$ $\mathrm{CaCl}_{2}$ ) and the interaction between calcium ion and the charged groups of the fiber. The binding constant $K_{\mathrm{b}}$ used in Fig. $4 \mathrm{a}$ is zero, and, therefore, this figure illustrates the fact that increasing the fraction of calcium chloride reduces the Donnan potential (i.e. reduces the electrostatic binding between the drug and the charged groups of the fiber) and increases the drug release. Fig. $4 \mathrm{~b}$ considers a moderate value of the binding constant, which could be the case in Smopex ${ }^{\circledR}-101$ and -107 fibers. In agreement with the experimental observations in Fig. 2a,c, Fig. 4b shows that increasing the fraction of calcium chloride increases the drug release. This is basically due to the reduction of the effective charge of the fiber, $z_{m} c_{m}$, due to the formation of $\mathrm{COO} \cdots \mathrm{Ca}^{+}$groups, which leads to a reversal of the sign of $z_{m} c_{m}$, when the fraction of $\mathrm{CaCl}_{2}$ is $90 \%$. For the lower values of the $\mathrm{CaCl}_{2}$ fraction, the effective fixed charge does not reverse the sign, but it is so small that the fiber behaves as an anion exchanger (i.e. $K_{\mathrm{e}}<1$ ) due to the high value of the drug lipophilicity considered, 
$K_{\mathrm{c}, \mathrm{d}}=300$ (see the comments after Eq. (9)). This behavior as an anion-exchange fiber explains the significant increase in drug release observed in Fig. 4b with respect to Fig. 4a. Finally, Fig. 4c considers the case of the strong binding of calcium ions to the carboxylic groups, which could be the case in Smopex ${ }^{\circledR}-102$ fiber. Once again, the agreement with experimental observations is quite good, and similarly to Fig. 2b, Fig. 4c shows that the trend of increasing drug release with the increasing fraction of $\mathrm{CaCl}_{2}$ is reversed between 50 and $90 \%$ of $\mathrm{CaCl}_{2}$. This is due to the fact that the amount of chloride ions increases with the increasing fraction of calcium chloride, even though the total concentration of the mixed solution is kept constant at $15 \mathrm{mM}$. The increasing amount of chloride ions is equivalent to the increasing concentration of the external solution (at a constant volume $V$ ) and, hence, makes the Donnan potential closer to 1 , which implies lower drug release in this case where the fiber behaves as an anion exchanger.

\section{Conclusions}

The present study has shown how the drug and fiber properties, and the concentration and nature of the external electrolyte solution affect the drug release from ion-exchange fibers. The experimental work has demonstrated that the release of ionic drugs loaded into ion-exchange fibers can be controlled by modifying either the fiber type or the external solution. The simulations of the experiments have yielded drug release values, which are quite similar to those observed experimentally, thus indicating that the theoretical modelling proposed describes adequately the basic trends of the behavior of these systems.

\section{Acknowledgements}

The financial support from TEKES (the Technology Development Centre in Finland) is gratefully acknowledged. J.A.M. thanks the DGICYT (Ministry of Education of Spain) for financial support through project No. PB98-0419. J.H. thanks also the Academy of Finland for financial support.

\section{References}

[1] A.K. Banga, Y.W. Chien, Hydrogel-based iontotherapeutic delivery devices for transdermal delivery of peptide/protein drugs, Pharm. Res. 10 (1993) 697-702.

[2] C.D. Ebert, W. Heiber, R. Andriola, P. Williams, Development of a novel transdermal system design, J. Controlled Release 6 (1987) 107-111.

[3] B.A. Adams, E.L. Holmes, Absorptive properties of synthetic resins. Part I, J. Soc. Chem. Ind. 54 (1935) 1T-6T.

[4] G. Burke, R. Mendes, S. Jambhekar, Investigation of the applicability of ion exchange resins as a sustained release drug delivery system for propranolol hydrochloride, Drug. Dev. Ind. Pharm. 12 (1986) 713-732.

[5] W.J. Irwin, R. MacHale, P.J. Watts, Drug-delivery by ionexchange. Part VII: release of acidic drugs from anionic exchange resinate complexes, Drug Dev. Ind. Pharm. 16 (1990) 883-898.

[6] R. Jani, O. Gan, Y. Ali, R. Rodstrom, S. Hancock, Ion exchange resins for ophthalmic delivery, J. Ocul. Pharmacol. 1 (1994) 57-67.

[7] O.M. Conaghey, J. Corish, O.I. Corrigan, The release of nicotine from a hydrogel containing ion exchange resins, Int. J. Pharm. 170 (1998) 215-224.

[8] O.M. Conaghey, J. Corish, O.I. Corrigan, Iontophoretically assisted in vitro membrane transport of nicotine from a hydrogel containing ion exchange resins, Int. J. Pharm. 170 (1998) 225-237.

[9] T. Jaskari, M. Vuorio, K. Kontturi, A. Urtti, J.A. Manzanares, J. Hirvonen, Controlled transdermal iontophoresis by ionexchange fiber, J. Controlled Release 67 (2000) 179-190.

[10] F. Helfferich, Ion Exchange, Dover, New York, 1995.

[11] Y. Raghunathan, L. Amsel, O. Hinsvark, W. Bryant, Sustained-release drug delivery system I: coated ion-exchange resin system for phenylpropanolamine and other drugs, J. Pharm. Sci. 70 (1981) 379-384.

[12] R.H. Guy, J. Hadgraft, Rate-control in transdermal drug delivery, Int. J. Pharm. 82 (1992) R1-R6.

[13] N. Laksminarayanaiah, Equations of Membrane Biophysics, Academic Press, Orlando, FL, 1984, Sections 3.4, 3.6, 3.7.

[14] J. Lyklema, Fundamentals of Colloid and Interface Science, Vol. 1, Academic Press, London, 1991, Section 2.20.

[15] T.S. Sørensen, S.R. Rivera, Electrochemical characterization of membranes and membrane surfaces by EMF measurements, in: T.S. Sørensen (Ed.), Surface Chemistry and Electrochemistry of Membranes, Marcel Dekker, New York, 1999, Chapter 10.

[16] W.N. Charman, D.P. Christy, E.P. Geunin, D.C. Monkhouse, Interaction between calcium, a model divalent cation, and a range of poly(acrylic acid) resins as a function of solution pH, Drug Dev. Ind. Pharm. 17 (1991) 271-280.

[17] S. Mafé, P. Ramírez, Ion equilibrium and transport in weak 
amphoteric membranes, in: T.S. Sørensen (Ed.), Surface Chemistry and Electrochemistry of Membranes, Marcel Dekker, New York, 1999, Chapter 12.

[18] O.L. Sprockel, J.C. Price, Evaluation of sustained release aqueous suspensions containing microencapsulated drugresin complexes, Drug Dev. Ind. Pharm. 15 (1989) 12751287.
[19] W.J. Irwin, K.A. Belaid, H.O. Alpar, Drug-delivery by ionexchange. Part III: interaction of ester pro-drugs of propranolol with cationic exchange resins, Drug Dev. Ind. Pharm. 13 (1987) 2047-2066. 